University of Wollongong

Research Online

Australian Institute for Innovative Materials -

Papers

Australian Institute for Innovative Materials

$1-1-2015$

Origin of the enhanced piezoelectric thermal stability in $\mathrm{BiScO} 3-\mathrm{PbTiO} 3$ single crystals

Lingping Kong

Center for High Pressure Science and Technology Advanced Research

Gang Liu

Center for High Pressure Science and Technology Advanced Research

Shujun Zhang

Pennsylvania State University, shujun@uow.edu.au

Wenge Yang

Carnegie Institute of Washington

Follow this and additional works at: https://ro.uow.edu.au/aiimpapers

Part of the Engineering Commons, and the Physical Sciences and Mathematics Commons

Research Online is the open access institutional repository for the University of Wollongong. For further information contact the UOW Library: research-pubs@uow.edu.au 


\title{
Origin of the enhanced piezoelectric thermal stability in BiSc03-PbTiO3 single crystals
}

\author{
Abstract \\ $\mathrm{BiScO} 3$ - $\mathrm{PbTiO} 3$ single crystals were reported to possess high piezoelectric coefficient of $1200 \mathrm{pC} / \mathrm{N}$ and \\ Curie temperature of $>400^{\circ} \mathrm{C}$, exhibiting excellent thermal stability of properties up to $350^{\circ} \mathrm{C}$. However, \\ the origin of the thermal stability is yet unclear. In this research, high resolution synchrotron-based \\ technique was used to study the temperature driven structural evolution in $\mathrm{BiScO} 3-\mathrm{PbTiO} 3$ system, \\ where two competing symmetries and local distortion were observed, accounting for the high \\ piezoelectric activity. A strong correlation between thermal stability of structure and temperature- \\ dependent properties was established, which will benefit the design of ferroelectric materials with broad \\ temperature usage range. \\ Disciplines \\ Engineering | Physical Sciences and Mathematics

\section{Publication Details} \\ Kong, L., Liu, G., Zhang, S. \& Yang, W. (2015). Origin of the enhanced piezoelectric thermal stability in \\ BiSc03-PbTiO3 single crystals. Applied Physics Letters, 106 (23), 232901-1-232901-5.
}




\section{Origin of the enhanced piezoelectric thermal stability in $\mathrm{BiScO}_{3}-\mathrm{PbTiO}_{3}$ single crystals}

Lingping Kong, Gang Liu, Shujun Zhang, and Wenge Yang

Citation: Appl. Phys. Lett. 106, 232901 (2015); doi: 10.1063/1.4922345

View online: https://doi.org/10.1063/1.4922345

View Table of Contents: http://aip.scitation.org/toc/apl/106/23

Published by the American Institute of Physics

\section{Articles you may be interested in}

High performance ferroelectric relaxor- $\mathrm{PbTiO}_{3}$ single crystals: Status and perspective Journal of Applied Physics 111, 031301 (2012); 10.1063/1.3679521

Ultrahigh strain and piezoelectric behavior in relaxor based ferroelectric single crystals Journal of Applied Physics 82, 1804 (1997); 10.1063/1.365983

Field induced domain switching as the origin of anomalous lattice strain along non-polar direction in rhombohedral $\mathrm{BiScO}_{3}-\mathrm{PbTiO}_{3}$ close to the morphotropic phase boundary

Applied Physics Letters 107, 052901 (2015); 10.1063/1.4927678

Dielectric relaxation and charged domain walls in $(\mathrm{K}, \mathrm{Na}) \mathrm{NbO}_{3}$-based ferroelectric ceramics Journal of Applied Physics 121, 074101 (2017); 10.1063/1.4975341

Coupled domain wall motion, lattice strain and phase transformation in morphotropic phase boundary composition of $\mathrm{PbTiO}_{3}-\mathrm{BiScO}_{3}$ piezoelectric ceramic

Journal of Applied Physics 120, 154104 (2016); 10.1063/1.4964947

High temperature limitation due to onset of depoling in $\mathrm{BiScO}_{3}-\mathrm{PbTiO}_{3}$

Journal of Applied Physics 121, 064106 (2017); 10.1063/1.4975785

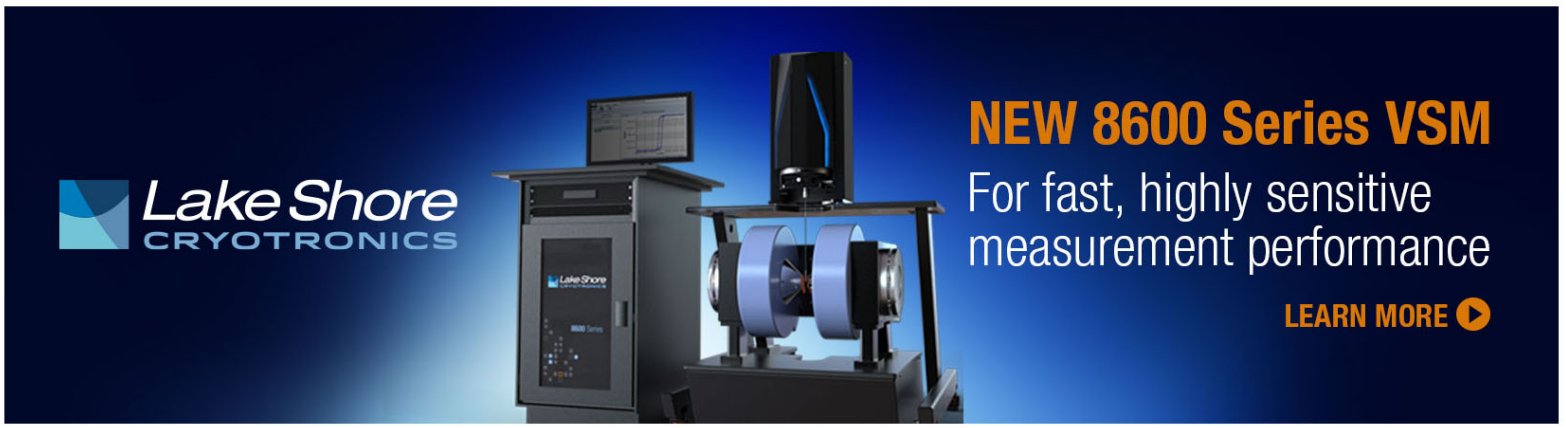




\title{
Origin of the enhanced piezoelectric thermal stability in $\mathrm{BiScO}_{3}-\mathrm{PbTiO}_{3}$ single crystals
}

\author{
Lingping Kong, ${ }^{1}$ Gang Liu, ${ }^{1, a)}$ Shujun Zhang, ${ }^{2, a)}$ and Wenge Yang ${ }^{1,3}$ \\ ${ }^{1}$ Center for High Pressure Science and Technology Advanced Research, Shanghai 201203, China \\ ${ }^{2}$ Materials Research Institute, The Pennsylvania State University, University Park, Pennsylvania 16802, USA \\ ${ }^{3}$ High Pressure Synergetic Consortium, Carnegie Institute of Washington, Argonne, Illinois 60439, USA
}

(Received 22 April 2015; accepted 25 May 2015; published online 8 June 2015)

\begin{abstract}
$\mathrm{BiScO}_{3}-\mathrm{PbTiO}_{3}$ single crystals were reported to possess high piezoelectric coefficient of $1200 \mathrm{pC} / \mathrm{N}$ and Curie temperature of $>400^{\circ} \mathrm{C}$, exhibiting excellent thermal stability of properties up to $350^{\circ} \mathrm{C}$. However, the origin of the thermal stability is yet unclear. In this research, high resolution synchrotron-based technique was used to study the temperature driven structural evolution in $\mathrm{BiScO}_{3}-\mathrm{PbTiO}_{3}$ system, where two competing symmetries and local distortion were observed, accounting for the high piezoelectric activity. A strong correlation between thermal stability of structure and temperature-dependent properties was established, which will benefit the design of ferroelectric materials with broad temperature usage range. (C) 2015 AIP Publishing LLC.
\end{abstract}

[http://dx.doi.org/10.1063/1.4922345]

Ferroelectric materials with perovskite structure are the mainstays for piezoelectric applications such as medical transducers, ultrasonic motors, underwater sonars, and sensors. ${ }^{1,2}$ Of particular interest is the relaxor- $\mathrm{PbTiO}_{3}$ single crystals with morphotropic phase boundary (MPB) compositions, have been actively studied for the last two decades due to their ultra-high piezoelectric coefficients and electromechanical coupling factors, being on the order of $2000 \mathrm{pC} / \mathrm{N}$ and 0.9 , far-outperforming the conventional piezoelectric ceramics. ${ }^{3-5}$ However, due to the relatively low Curie temperature and ferroelectric phase transition temperature, there is a long-term challenge for using these high-performance piezocrystals without sacrificing the temperature stability and depoling at elevated temperatures, thus, material systems with higher Curie temperature are desired. ${ }^{6,7}$ However, materials with higher Curie temperature generally possessing lower piezoelectric properties, the suppression of piezoelectric activity concurrent with elevated phase transition temperature is associated with a hard "polarization extension" effect, which can be explained using the phenomenological theory. ${ }^{8}$ Fig. 1 summarizes the piezoelectric coefficients of various perovskite-type ferroelectric polycrystalline ceramics and single crystals as a function of Curie temperature, among all high performance piezoelectrics with $d_{33}>1000 \mathrm{pC} / \mathrm{N}, \mathrm{BiScO}_{3^{-}}$ $\mathrm{PbTiO}_{3}$ (BSPT) single crystals were found to possess the highest Curie temperature of $>400^{\circ} \mathrm{C}$, thus, they are promising candidates for high-performance and high-temperature electroacoustic devices.

MPB in BSPT solid solutions is far away from being fully understood and there are still debates about the actual symmetry of the phases due to the following reasons: (1) the structure of ferroic solid solution is sensitive to many microstructural effects, including the intergranular strain/stress, grain size, local cation order, and chemical homogeneity; ${ }^{12}$ and (2) the difference and/or distortion in the structure of the

\footnotetext{
${ }^{\text {a) }}$ Authors to whom correspondence should be addressed. Electronic addresses: hit071202@gmail.com (Gang Liu) and soz1@psu.edu (Shujun Zhang)
}

two competing phases are too subtle to be fully resolved by ordinary x-ray techniques. ${ }^{13}$ The phase diagram of BSPT was first proposed by Eitel et al., that introducing a $R 3 m$ (rhombohedral)-P4 mm (tetragonal) MPB,${ }^{14}$ and then revised by Chaigneau et al. ${ }^{15}$ with emphasis on the coexistence of $\mathrm{Cm}$ (monoclinic) and $\mathrm{P} 4 \mathrm{~mm}$ symmetries, which has been argued and suggested to be replaced by either two orthorhombic or two monoclinic lattices. ${ }^{16}$ However, it is still a question that whether a tetragonal phase is inherent or should be excluded from the MPB region of BSPT system, since this symmetry has been observed in polycrystalline ceramics and nanocrystals. ${ }^{17-19}$ These open issues motivate the study of the symmetries in BSPT single crystals and their temperature-driven evolutions, to explore the origin of high piezoelectric activity and the excellent temperature stability of functionality. In this study, samples with single crystal form were studied to eliminate or at least significantly reduce the microstructure effects associated with polycrystalline ceramics. Synchrotron-based technology with high $d$-spacing

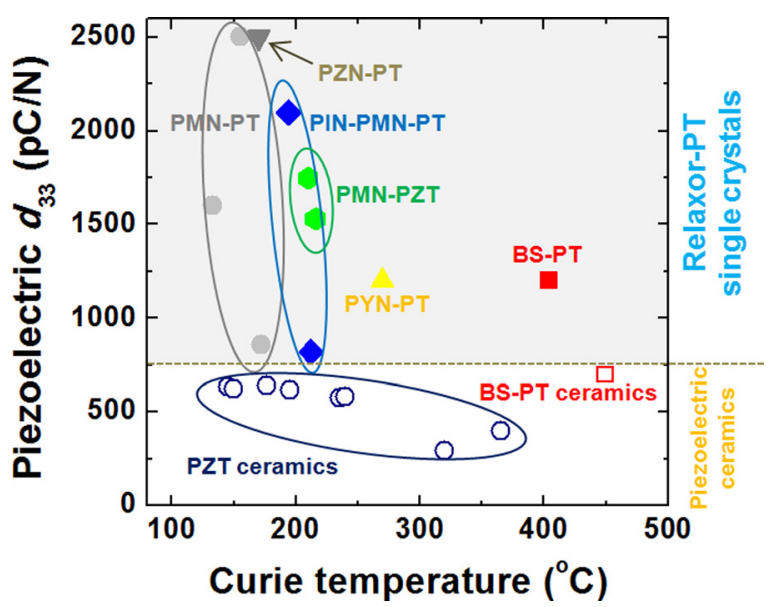

FIG. 1. The relationship between Curie temperature and longitudinal piezoelectric constant $d_{33}$ for various relaxor-PT single crystals and polycrystalline piezoceramics. Data are from Refs. 4 and 9-11. 
resolution together with dielectric measurement and Raman spectroscopy were employed to carefully resolve the symmetries as a function of temperature. The resolved structure is expected to benefit the design of high performance piezoelectric materials with high Curie temperature, and provide us the key to fundamentally understand the applications of relaxor-PT materials at elevated temperature conditions.

High quality $0.37 \mathrm{BiScO}_{3}-0.63 \mathrm{PbTiO}_{3}$ (BSPT37/63) single crystals were grown using the high temperature solution method. The samples for XRD measurements were annealed at $600{ }^{\circ} \mathrm{C}$ to remove residual stress. In-situ synchrotron XRD experiments were performed at Argonne National Laboratory beam line 11-BM, at which the instrument resolution is $\Delta d / d$ $\sim 0.00017$, representing the state-of-the-art $d$-spacing resolution for diffraction measurements. ${ }^{20}$ Rietveld refinements of XRD data were performed using GSAS program. The dielectric properties as a function of temperature from room temperature to $480{ }^{\circ} \mathrm{C}$ were measured using $\mathrm{HP} 4284 \mathrm{~A}$ precision LCR meter connected to a computer controlled temperature chamber. Raman spectroscopy measurements were performed from room temperature up to $460^{\circ} \mathrm{C}$ using a $514.5 \mathrm{~nm}$ excitation laser source.

In-situ synchrotron XRD was employed on BSPT37/63 at low temperature of $100 \mathrm{~K}\left(-173^{\circ} \mathrm{C}\right)$. The diffraction profile around pseudocubic $\{111\}_{\mathrm{pc}}$ diffraction peak was given in Fig. 2(a), multi-peak characteristic was observed where four peaks can be resolved, indicating that the obtained XRD pattern cannot be satisfied by any single space group model, including $R 3 \mathrm{~m}, P 4 \mathrm{~mm}, \mathrm{Pm}$, and $\mathrm{Cm}$, since these space groups only allow for no more than three $\{111\}_{\text {pc }}$ peaks. The crystallographic refinements for the diffraction pattern were then performed. As expected, a single model cannot fully describe the measured diffraction pattern, which explains the high $\mathrm{R}_{\mathrm{wp}}$ and $\mathrm{R}_{\mathrm{p}}$ values (two parameters between theoretical and experimental XRD patterns, which can be used to monitor the convergence of the structure model), being on the order of $>9.6 \%$ and $>7.35 \%$, respectively. ${ }^{21}$ Four mixphase models, $R 3 m+P m, R 3 m+C m, P m+P 4 m m$, and
$\mathrm{Cm}+\mathrm{P} 4 \mathrm{~mm}$, were further considered. It was found that the fitting quality of the refinement using $\mathrm{Cm}+\mathrm{P} 4 \mathrm{~mm}$ set (Fig. 2(b)) is superior to another three models, ${ }^{21}$ reflected by the relatively small $\mathrm{R}_{\mathrm{wp}}(6.24 \%)$ and $\mathrm{R}_{\mathrm{p}}(4.90 \%)$, in good agreement with the previous reported BSPT structure in polycrystalline ceramics and nanocrystals. ${ }^{17-19}$ Due to the larger number of the refined structural parameters, it is possible to fit the pattern to two individual monoclinic phases or three coexisted phases. However, $\mathrm{Cm}+\mathrm{P} 4 \mathrm{~mm}$ model is most likely to accurately represent the real material structure of the studied sample since: (1) BSPT37/63 is located at tetragonal-rich MPB, in which the tetragonal phase cannot be completely ignored; (2) $\mathrm{Cm}$ is associated with one of the three monoclinic phases, i.e., $\mathrm{M}_{\mathrm{A}}$; and (3) by expanding Landau free energy to six orders, it is demonstrated that $\mathrm{M}_{\mathrm{A}}$ - $\mathrm{T}$ is a favorable polarization rotation path, indicating a delicate balance between these two phases, ${ }^{2,23}$ accounting for the observed phases coexistence.

To investigate the phase transition sequence and temperature-driven structural evolution, XRD patterns were collected from -173 to $500{ }^{\circ} \mathrm{C}$ and selected Bragg reflections was shown in Fig. 2(c). The low temperature phases of $\mathrm{Cm}+\mathrm{P} 4 \mathrm{~mm}$ were initially used to model patterns at evaluated temperatures and it was found such a structure persisted up to $360^{\circ} \mathrm{C}$, at which the two phases merged to a single $P 4 \mathrm{~mm}$, associated with a $\mathrm{Cm}+P 4 \mathrm{~mm} \rightarrow P 4 \mathrm{~mm}$ ferroelectric phase transition, followed by a $P 4 m m \rightarrow P m \overline{3} m$ phase transition above $440^{\circ} \mathrm{C}$ (Fig. 2(d)). The temperature dependences of the lattice parameters as well as the fractions of the monoclinic phase and monoclinic angles were given in Figs. 2(e) and 2(f), respectively. The two competing phases establish a tetragonal-rich MPB, at which an easy polarization rotation and facilitated domain wall/interphase boundary motions are expected to contribute to an enhanced functionality.

Fig. 3(a) shows the Raman spectra of BSPT37/63 taken from room temperature to $460{ }^{\circ} \mathrm{C}$. The Raman peak positions as a function of temperature are determined and given in

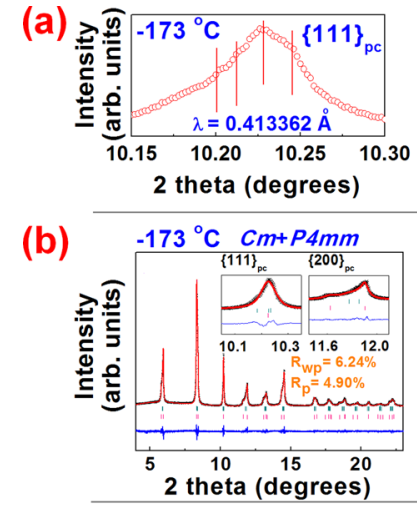

(e)

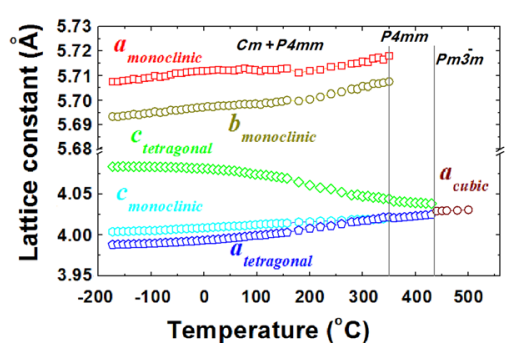

(f)
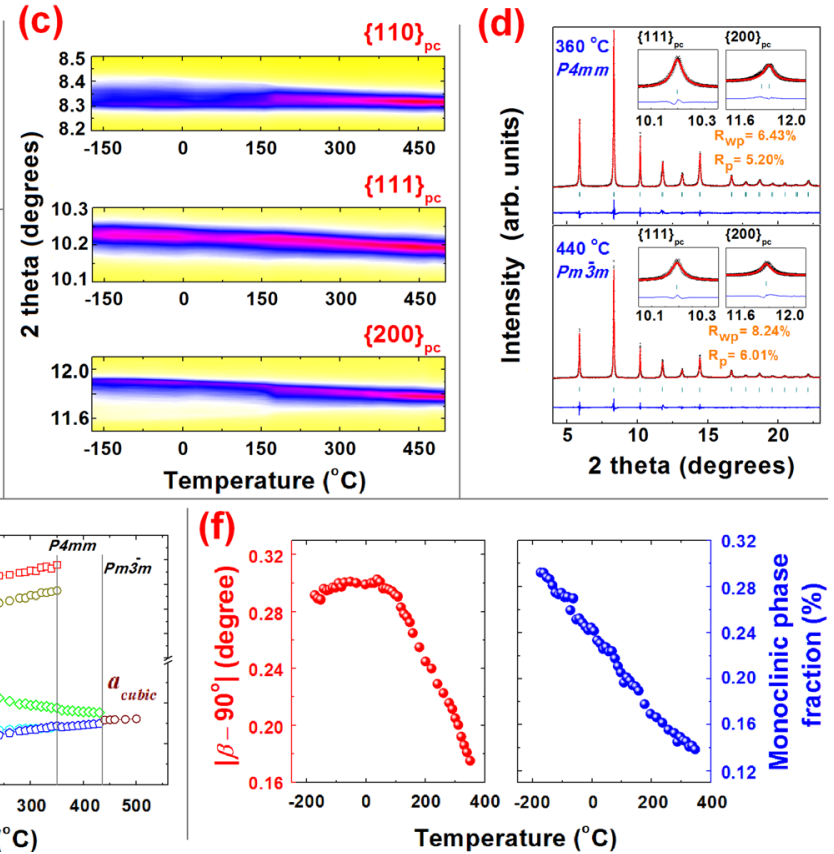

FIG. 2. High resolution synchrotron XRD investigations on BSPT37/63: (a) XRD profile for $\{111\}_{\mathrm{pc}}$ reflections of BSPT37/63 crystals collected at $-173{ }^{\circ} \mathrm{C}$ (the superscript "pc" denotes pseudocubic unit cell). Four resolved peaks are highlighted by red lines; (b) Rietveld refinement of the XRD pattern measured at $-173{ }^{\circ} \mathrm{C}$ using $\mathrm{Cm}$ + $\mathrm{P} 4 \mathrm{~mm}$ model; (c) Selected Bragg reflections from -173 to $500{ }^{\circ} \mathrm{C}$; (d) Rietveld refinement of the patterns measured at $360^{\circ} \mathrm{C}$ and $440{ }^{\circ} \mathrm{C}$ using $P 4 \mathrm{~mm}$ and $P m \overline{3} \mathrm{~m}$ models, respectively; (e) Temperature dependence of lattice constants; and (f) Temperature dependence of $\left|\beta-90^{\circ}\right|$ angle and fraction of monoclinic phase. 
(a)

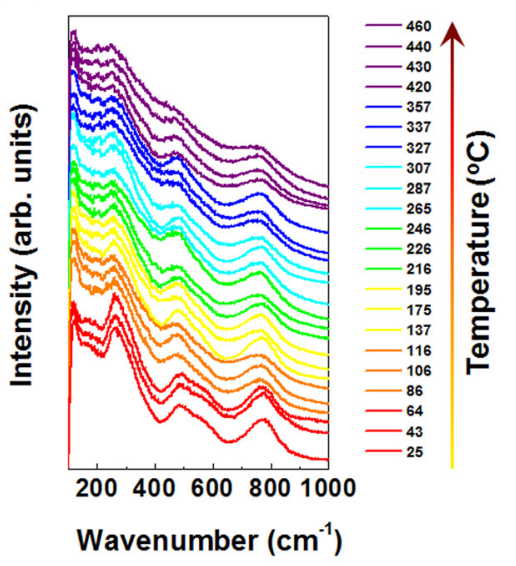

(b)

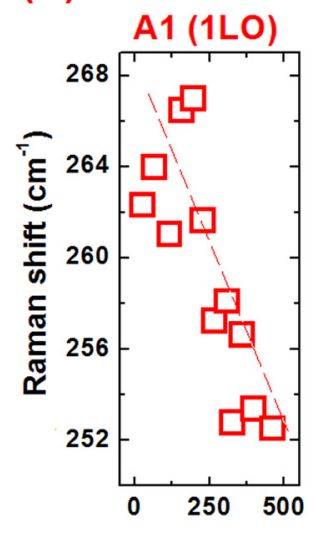

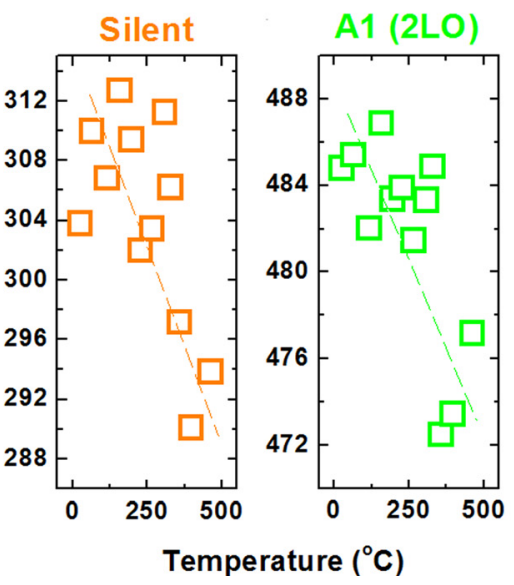

A1 (3TO)

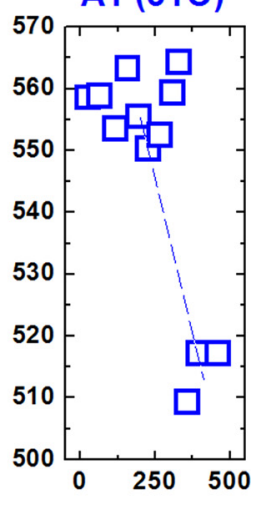

FIG. 3. (a) Temperature-dependent Raman spectra performed on BSPT37/63 crystals from room temperature to $460{ }^{\circ} \mathrm{C}$; and (b) Temperature dependence of the four Raman bands. The dashed lines are the eye guidelines, indicating the softening of the lattice vibration and the phase transition.

Fig. 3(b), in which the A1(1LO) and silent modes reflect the Ti-O/Sc-O bending and/or stretching, while the A1(2LO) and $\mathrm{A} 1(3 \mathrm{TO})$ correspond to the titling of $\mathrm{TiO}_{6} / \mathrm{ScO}_{6}$ octahedra. ${ }^{24}$ All four modes move to low frequency region as temperature increases, suggesting a softening of the lattice vibration, similar to that observed in BSPT nano-powders. ${ }^{25}$ There is an abrupt decrease in Raman shift near $360^{\circ} \mathrm{C}$ for the two high-frequency modes A1(2LO) and A1(3TO), which can be explained by monoclinic $\rightarrow$ tetragonal phase transition. All peaks are relatively broad compared to classic ferroelectrics $\mathrm{PbTiO}_{3}$, being associated with the band overlapping, demonstrating a typical characteristic of relaxor ferroelectrics. Such a local disorder can further make the local structure deviate from a perfect cubic structure above Curie temperature, and thus allow Raman signatures to remain active even at high temperature. ${ }^{26}$

Dielectric permittivity and loss of BSPT37/63 were investigated from room temperature to $480^{\circ} \mathrm{C}$. As shown in Fig. 4, two dielectric anomalies were observed at $\sim 360$ and $\sim 440^{\circ} \mathrm{C}$, which were assumed to be the monoclinic $\rightarrow$ tetragonal phase transition and Curie temperatures, respectively, in good agreement with the XRD data. The relaxor behavior of BSPT37/63 was investigated, as given in the inset of Fig. 4, the degree of diffuseness $(\gamma)$ value was found to be between 1.555 and 1.622, indicating that BSPT37/63 crystals maintain a relaxor-like behavior, ${ }^{27}$ being consistent

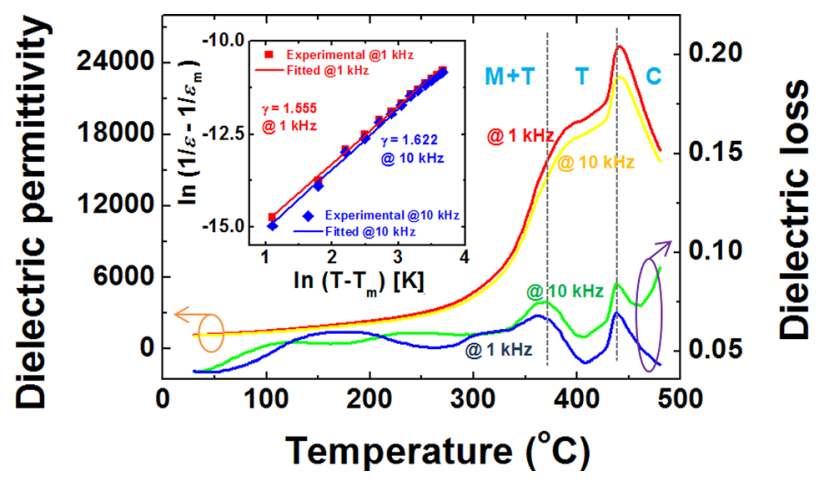

FIG. 4. Temperature dependences of dielectric permittivity and dielectric loss (measured at $1 \mathrm{kHz}$ and $10 \mathrm{kHz}$ ) for [001] c poled BSPT37/63 crystals, in which the inset shows the fitted relaxor behavior by modified Curie law. with the observed Raman results. The dielectric permittivity is nearly temperature independent in the range of room temperature to $300{ }^{\circ} \mathrm{C}$, being on the order of $<10 /{ }^{\circ} \mathrm{C}$, together with the high electromechanical and piezoelectric properties reported previously, demonstrating enhanced thermal stability of functionality in BSPT crystals.

To understand the origin of thermal stability of properties, the temperature-dependent $c / a$ and $\left|\beta-90^{\circ}\right|$ of various relaxorPT crystals were summarized in Figs. 5(a) and 5(b), respectively. Here, $c / a$ is the ferroelectric tetragonality and $\left|\beta-90^{\circ}\right|$ is used to indicate how much the monoclinic unit cell deviates from $90^{\circ}$. Compared to other relaxor-PT counterparts, both parameters of BSPT were relatively stable with respect to temperature. The tetragonality $c / a$ was found to decrease by about only 0.02 upon raising the temperature from -173 to $400{ }^{\circ} \mathrm{C}$, whereas the $\left|\beta-90^{\circ}\right|$ value was less than $0.1^{\circ}$ from -173 to $300^{\circ} \mathrm{C}$, indicating the enhanced structural stability in both tetragonal and monoclinic phases. Based on a simplest rigid model, ${ }^{31,32}$ in ionic crystals, the potential energy of the ion pair with $+q$ and $-q$ ions can be written as follows:

$$
\Delta U=U(r)-U\left(r_{0}\right)=f\left(r-r_{0}\right)^{2}-g\left(r-r_{0}\right)^{3} .
$$

Here, $r$ is the distance between a cation and its nearest neighbor anion and $r_{0}$ is the equilibrium position. Then the electrostrictive coefficient $Q_{11}$ is given as follows:

$$
Q_{11}=12 g r_{0}^{5} / f q^{2}
$$
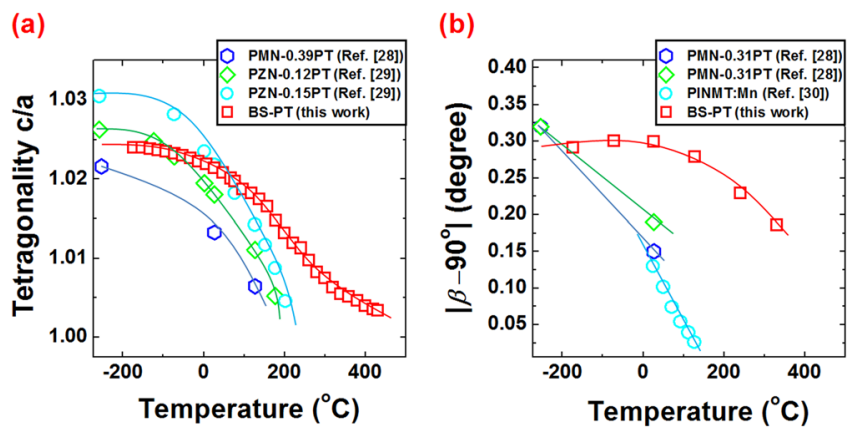

FIG. 5. Temperature dependences of (a) tetragonal ferroelectricity $c / a$; and (b) monoclinic angle $\left|\beta-90^{\circ}\right|$ for various relaxor-PT crystals. Data are from Refs. 28-30 and this work. 
Note that the coefficients $f$ and $g$ in Eqs. (1) and (2) are associated with the crystal structure as well as the charge and radius of ions, ${ }^{31,32}$ thus the strong temperature stability of crystal structure observed in BSPT system (Fig. 5) demonstrates the thermally stable electrostrictive coefficients $Q$. In addition, it is well known that spontaneous polarization $P_{S}$ is defined by the value of electric dipole moment per unit volume which can be written as follows:

$$
P_{S}=\frac{\langle p\rangle}{V},
$$

where $V$ is the volume and $p$ is the electrical dipole. For a unit cell, $V$ is determined by the crystal lattice parameter and $p$ can be written as follows:

$$
p=q r_{0}
$$

where $r_{0}$ is the equilibrium distance between cation and anion that strongly associated with the crystal structure. Compared to other relaxor-PT counterparts, these structural parameters in BSPT system were found to maintain similar values at elevated temperatures, thus a high thermal stability of the spontaneous polarization $P_{S}$ can be expected. In the framework of the thermodynamic theory, ${ }^{33}$ the piezoelectric coefficients of perovskite ferroelectrics can be expressed in terms of dielectric permittivity $\varepsilon$, spontaneous polarization $P_{S}$, and electrostrictive coefficients $Q$. Taking $P 4 \mathrm{~mm}$ symmetry as an example, the longitudinal piezoelectric coefficients can be written as follows:

$$
d_{33}=2 \varepsilon_{33}^{\mathrm{T}} Q_{11} P_{3} .
$$

Therefore, considering the nearly temperature-independent characteristic of dielectric properties (see Fig. 4), enhanced thermal stability of piezoelectric properties of BSPT system
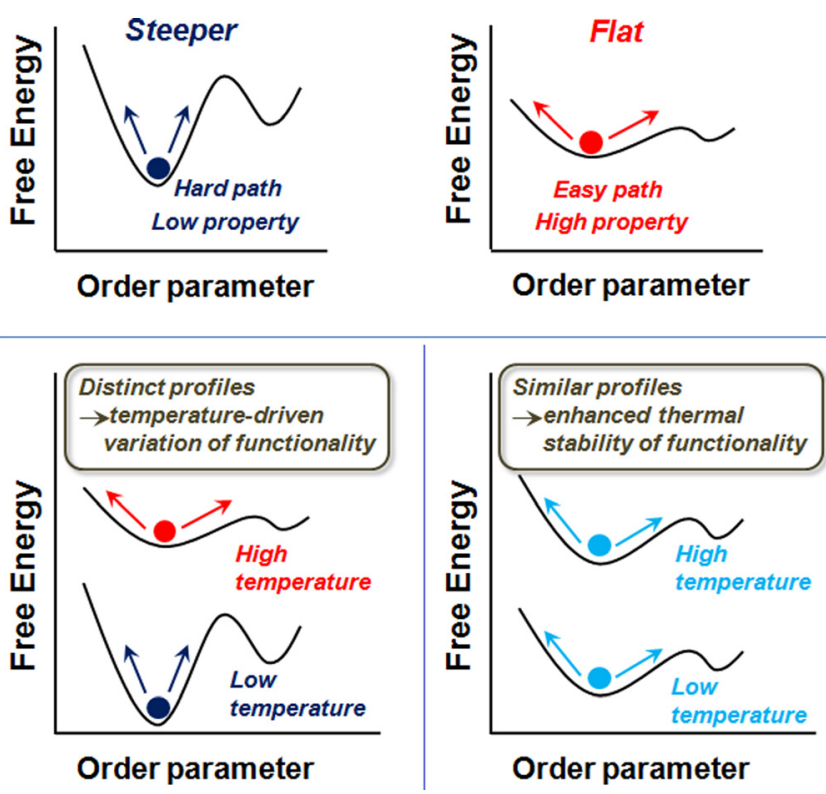

FIG. 6. Schematic represents relationship between free energy profile and thermal stability of functionality. Top: flat energy profile is associated with superior functionality; Bottom left: obvious difference in energy profiles at low and high temperatures suggests an obvious variation of properties driven by temperature; and Bottom right: the enhanced thermal stability is concurrent with the temperature-independent or similar energy profiles at low and high temperatures. can be understood. In general, from Landau-GinzburgDevonshire theory on phase transitions, the free energy of the system can be expanded in a power series of the order parameter $\varphi$ as follows: ${ }^{34}$

$$
F=F_{0}+\sum_{n} \frac{1}{n} \alpha_{n} \varphi^{n} .
$$

Here, $\varphi$ is usually nonzero below critical point and zero for high-symmetry phase. For tetragonal and monoclinic phases, $\frac{c}{a}-1$ and $\left|\beta-90^{\circ}\right|$ can be used as order parameters and free energy of tetragonal and monoclinic systems can be expanded in terms of them, respectively. Based on the results of temperature-driven evolution of the order parameters given in Figs. 5(a) and 5(b), the difference in free energy profile of BSPT at low and high temperatures should be much less than those of other relaxor-PT systems with thermally unstable structure. Therefore, the intrinsic piezoelectric activity, ${ }^{35}$ electrometrical coupling, ${ }^{35}$ and energy losses ${ }^{11}$ will exhibit thermal stable characteristics since they are strongly associated with the polarization variation and flatness of the potential wells described by free energy profile, as plotted in Fig. 6. The proposed model helps us to understand the difference between BSPT and other relaxor-PT systems, while for a given system the compositions far away from the MPB possess higher thermal stability and field stability, implying the importance of phase transition. As approaching to the MPB, due to the more aggressive competing between phases separated by phase boundary, the crystal structure will be unstable under weak external stimuli including small temperature variation. Consequently, at low and high temperatures, there must be obvious difference in structurally related parameters such as electrostrictive coefficients $Q$ and spontaneous polarization $P_{S}$, thus the thermal instability of piezoelectricity can be explained.

In summary, the thermal stability of ferroelectricity for $\mathrm{BiScO}_{3}-\mathrm{PbTiO}_{3}$ single crystals was studied based on the structural evolution. The coexistence of two competing symmetries $\mathrm{P} 4 \mathrm{~mm}$ and $\mathrm{Cm}$ together with the local disorder characteristic are believed to be strongly associated with the high piezoelectric activity. Further analysis on the temperaturedriven variation of the ferroelectric order parameters revealed that the structural stability played a critical role in achieving thermally stable functionality.

The authors specially thank Dr. Matthew R. Suchomel for experimental help and useful discussions. Use of the Advanced Photon Source was supported by the U.S. Department of Energy, Office of Science, Office of Basic Energy Sciences, under Contract No. DE-AC02-06CH11357.

${ }^{1}$ S. E. Park and T. R. Shrout, J. Appl. Phys. 82, 1804 (1997).

${ }^{2}$ S. J. Zhang, F. Li, X. N. Jiang, J. Kim, J. Luo, and X. C. Geng, Prog. Mater. Sci. 68, 1 (2015).

${ }^{3}$ E. Sun and W. Cao, Prog. Mater. Sci. 65, 124 (2014).

${ }^{4}$ A. Gruverman and A. Kholkin, Rep. Prog. Phys. 69, 2443 (2006).

${ }^{5}$ S. J. Zhang and F. Li, J. Appl. Phys. 111, 031301 (2012).

${ }^{6}$ R. C. Turner, P. A. Fuierer, R. E. Newnham, and T. R. Shrout, Appl. Acoust. 41, 299 (1994).

${ }^{7}$ D. Damjanovic, Curr. Opin. Solid State Mater. Sci. 3, 469 (1998).

${ }^{8}$ D. Damjanovic, Appl. Phys. Lett. 97, 062906 (2010). 
${ }^{9}$ S. J. Zhang, C. A. Randall, and T. R. Shrout, Appl. Phys. Lett. 83, 3150 (2003).

${ }^{10}$ T. T. Zou, X. H. Wang, H. Wang, C. F. Zhong, L. T. Li, and I.-W. Chen, Appl. Phys. Lett. 93, 192913 (2008).

${ }^{11}$ G. Liu, S. J. Zhang, W. H. Jiang, and W. W. Cao, Mater. Sci. Eng., R 89, 1 (2015).

${ }^{12}$ B. Nehoda, Curr. Opin. Solid State Mater. Sci. 6, 27 (2002).

${ }^{13}$ B. Nehoda and D. E. Cox, Phase Transitions 79, 5 (2006).

${ }^{14}$ R. E. Eitel, S. J. Zhang, T. R. Shrout, C. A. Randall, and I. Levin, J. Appl. Phys. 96, 2828 (2004).

${ }^{15}$ J. Chaigneau, J. M. Kiat, C. Malibert, and C. Bogicevic, Phys. Rev. B 76, 094111 (2007).

${ }^{16}$ K. Datta, S. Gorfman, and P. A. Thomas, Appl. Phys. Lett. 95, 251901 (2009).

${ }^{17}$ T. Hungría, F. Houdellier, M. Algueró, and A. Castro, Phys. Rev. B 81, 100102(R) (2010).

${ }^{18}$ J. L. Jones, E. Aksel, G. Tutuncu, T.-M. Usher, J. Chen, X. R. Xing, and A. J. Studer, Phys. Rev. B 86, 024104 (2012).

${ }^{19}$ K. V. Lalitha, A. N. Fitch, and R. Ranjan, Phys. Rev. B 87, 064106 (2013).

${ }^{20}$ P. L. Lee, D. Shu, M. Ramanathan, C. Preissner, J. Wang, M. A. Beno, R. B. Von Dreele, L. Ribaud, C. Kurtz, S. M. Antao, X. Jiao, and B. H. Toby, J. Synchrotron Radiat. 15, 427 (2008).

${ }^{21}$ See supplementary material at http://dx.doi.org/10.1063/1.4922345 for GSAS refinement results using various models.
${ }^{22}$ H. Fu and R. E. Cohen, Nature 403, 281 (2000).

${ }^{23}$ D. Vanderbilt and M. H. Cohen, Phys. Rev. B 63, 094108 (2001).

${ }^{24}$ J. A. Lima, W. Paraguassu, P. T. C. Freire, A. G. Souza Filho, C. W. A. Paschoal, J. Mendes Filho, A. L. Zanin, M. H. Lente, D. Garcia, and J. A. Eiras, J. Raman Spectrosc. 40, 1144 (2009).

${ }^{25}$ S. P. Zhang, X. H. Wang, and L. T. Li, Ferroelectrics 450, 28 (2013).

${ }^{26}$ M. Ahart, R. E. Cohen, V. Struzhkin, E. Gregoryanz, D. Rytz, S. A. Prosandeev, H.-k. Mao, and R. J. Hemley, Phys. Rev. B 71, 144102 (2005).

${ }^{27}$ K. Uchino and S. Nomura, Ferroelectrics 44, 55 (1982).

${ }^{28}$ B. Noheda, D. E. Cox, G. Shirane, J. Gao, and Z.-G. Ye, Phys. Rev. B 66, 054104 (2002).

${ }^{29}$ D. La-Orauttapong, B. Noheda, Z.-G. Ye, P. M. Gehring, J. Toulouse, D. E. Cox, and G. Shirane, Phys. Rev. B 65, 144101 (2002).

${ }^{30}$ L. P. Kong, G. Liu, S. J. Zhang, and H. Z. Liu, J. Appl. Phys. 114, 144106 (2013).

${ }^{31}$ K. Uchino, S. Nomura, K. Vedam, R. E. Newnham, and L. E. Cross, Phys. Rev. B 29, 6921 (1984).

${ }^{32}$ F. Li, L. Jin, Z. Xu, and S. J. Zhang, Appl. Phys. Rev. 1, 011103 (2014).

${ }^{33}$ M. J. Haun, E. Furman, S. J. Jang, and L. E. Cross, Ferroelectrics 99, 13 (1989).

${ }^{34}$ J. Guyonnet, Ferroelectric Domain Walls: Statics, Dynamics, and Functionalities Revealed by Atomic Force Microscopy (Springer, Switzerland, 2014).

${ }^{35}$ D. Damjanovic, J. Am. Ceram. Soc. 88, 2663 (2005). 